\title{
Reconstruction of solar activity trends by using geomagnetic proxies
}

\author{
Dmitri I. Ponyavin \\ Institute of Physics, University of St.Petersburg, St.Petersburg, 198504, Russia, Email: \\ ponyavin@geo.phys.spbu.ru
}

\begin{abstract}
Geomagnetic activity variations are closely related to the global magnetic field of the Sun and could serve as useful proxies to reconstruct the solar wind history and evolution of the inner heliosphere. Historical geomagnetic records reveal trends in the solar wind and interplanetary magnetic field variability resulting from topological changes of the heliospheric current sheet and solar corona viewed from the Earth. Predictions of the solar cycle based on geomagnetic trends are presented.
\end{abstract}

\section{Sunspot numbers}

Sunspots are remarkable features at the Sun and present a unique opportunity to study long-term evolution of the solar activity. Rudolf Wolf in 1848 derived a simple measure of solar activity by counting a number of sunspots and sunspot groups at the solar disk. It was a time when nothing was known about magnetic fields of the Sun and latitude shift of occurrence rate of sunspots during a course of solar cycle (Butterfly diagram). However, in spite of long history of sunspots observations in the past, sunspot counts do not provide sufficient information about solar cyclicity and evolution of the global heliosphere. Following magnetic polarity rule, consecutive 11-year solar cycles tend to group and form a 22-year magnetic sunspot cycle. Moreover, each solar cycle starts when the first sunspots of the next 11-year cycle appear at high solar latitudes and the current 11-year cycle still progress in such a way that consecutive solar cycles overlap each other.

Therefore, sunspot counts only partially reflect a global organization of magnetic activity of the Sun. Time-series of sunspot numbers are a priori a poor convolution of distributed dynamical system and could not be predicted correctly using even embedding techniques. In the case when using the sunspot counts only, we face with the lack of sufficient information about the global processes responsible for the sunspot cyclicity and should use any additional characteristics to describe and predict correctly the sunspot activity behavior. Moreover, the inner heliosphere is formed basically by large-scale magnetic fields of the Sun not affected by sunspots.

\section{Geomagnetic variations}

Alexander Ohl first in 1966 used historical geomagnetic observations to predict sunspot activity. Following the Ohl' scheme consecutive sunspot and geomagnetic cycles are statistically interrelated. Each pair of the consecutive sunspot cycles begins with the even cycle which is somewhat usually lower than the odd sunspot cycle.

Low level of geomagnetic activity during the odd cycles precedes the relatively low sunspot maximum of the even cycles. The high geomagnetic level during the even cycles precedes the high sunspot maximum of the odd cycles, correspondingly (Ponyavin (2002)). Historical proxies on geomagnetic variations provide additional opportunity to study solar activity in the past and predict the future cycles. 


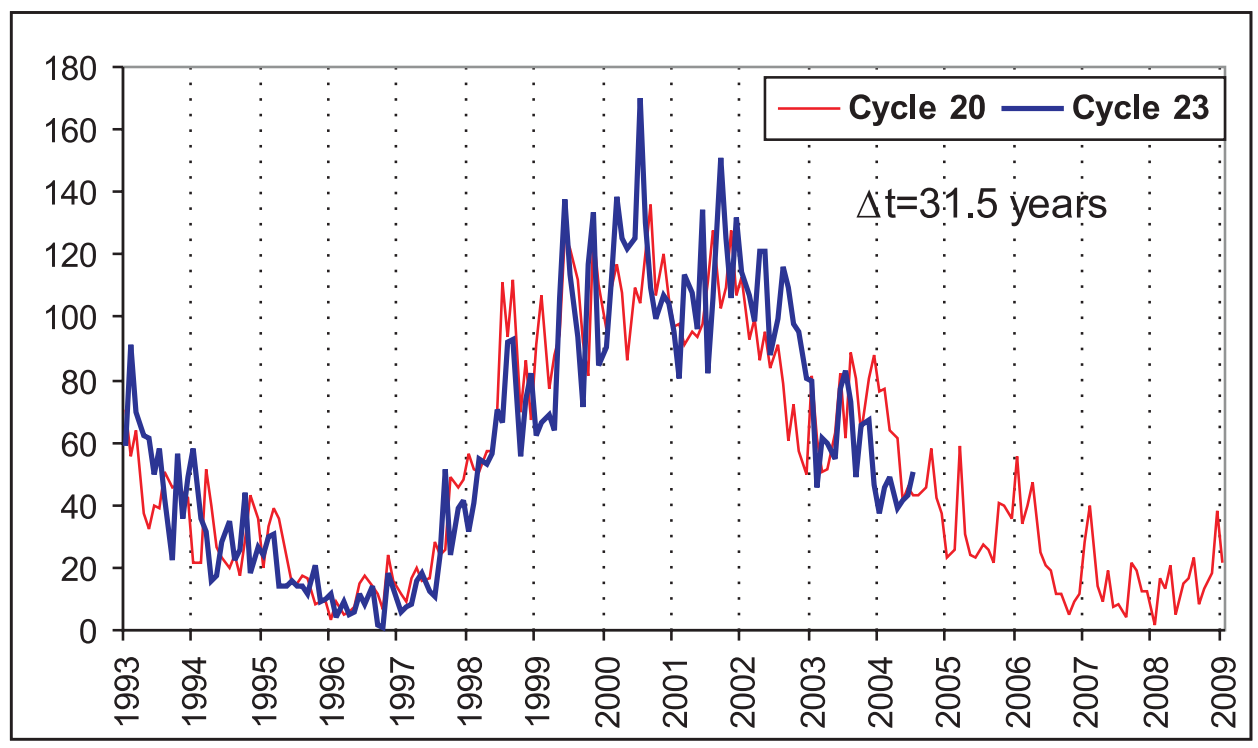

Figure 1. Sunspot numbers (monthly means) for the solar cycles 20 and 23 . Time shifting of the data corresponds to 31.5 years. The last point of solid line refers to July of 2004.

Geomagnetic variations reflect the solar wind and interplanetary magnetic field structure controlled by large-scale magnetic fields on the Sun. Geomagnetic storms coincides by time with enhancements of solar magnetic flux. Storms also correspond to periods of highest inclination of the heliospheric current sheet with respect to ecliptic plane. This situation can be interpreted in terms of non-axisymmetrical dipole-like magnetic filed of the Sun that changes drastically the solar coronal shape and produces powerful geomagnetic storms (Ponyavin (2001)).

\section{Forecasting}

Heliosphere exhibits long-term oscillations that can be used in forecasting. We have tried to predict the current solar cycle behavior using long-term quasi-periodicity persisted in the geomagnetic data. Edward Bruckner discovered in 1890 three-solar-cycle climate oscillation based on direct and proxy data (cf. Clough (1905)). Ahluwalia (1998) has reviewed the recent data for geomagnetic indices. These data indicate the presence of the long-term periodicity for the last 60 years.

In accordance with the Bruckner cycle we have shifted the sunspot data (monthly means) to compare solar cycles 20 and 23. Interesting is, that two curves even in details correspond each other. It also follows from the Figure 1 that the minimum of solar activity was expected in 2008.

\section{References}

Ahluwalia H.S. 1998 J. Geophys. Res., 103, 12103-12109.

Clough H.W. 1905 Astrophys.J., 22, 42-75.

Ponyavin D.I. 2001 Space Sci. Rev., 97, 225-228.

Ponyavin D.I. 2002 Adv. Space. Res., 29, 421-426. 